\title{
Diabetic Nephropathy Foreword
}

Received March 31, 2021

Accepted April 08, 2021

Jianghua Chen

Kidney Disease Center, The First Affiliated Hospital, Zhejiang University, College of Medicine, Hangzhou, Zhejiang Province, Chin

In the Twenty-first Century, social development has dramatically changed the spectrum of human diseases, and chronic non-communicable diseases have become one of the important public health problems threatening human health. Epidemiologically, $86.6 \%$ of the deaths in China (2015) were the result of chronic diseases and the burden caused by chronic disease accounted for more than $70 \%$ of the total disease burden (Progress in China's Disease Control and Prevention, 2015). Chronic kidney disease (CKD) has been established as one of the key chronic diseases for national prevention and control due to its high incidence, lots of complications, high medical costs, and high mortality. The authoritative epidemiological survey published in LANCET in 2012 showed that the prevalence rate of CKD in China was up to $10.8 \%$. Based on these data, the number of CKD patients in our country has exceeded 119.5 million and the number of uremia patients was about 1.5-2 million, which causes heavy medical, health, and economic burdens [1].

In the past, glomerulonephritis was considered the main cause of CKD in China, as shown by epidemiological data. However, in recent years, diabetic nephropathy has become an important cause of CKD, following the etiological spectrum of changes that have been occurring in relation to CKD. According to a report published in N Engl $J$ Med by researchers from Peking University First Hospital in China, diabetes has, since 2011, become the leading cause of CKD among urban residents and diabetic kidney damage accounts for a higher proportion of inpatients than glomerulonephritis. Further, diabetic nephropathy is one of the most common microvascular complications of diabetes, the incidence of which exceeds $50 \%$. Clinical data of 32,208 patients with type 2 diabetes in 33 countries around the world showed that $50 \%$ of patients with type 2 diabetes have kidney damage. Among these patients, $30-50 \%$ have elevated urine protein, and about $20 \%$ have an abnormally low glomerular filtration rate (GFR), i.e., a GFR that is $<60 \mathrm{~mL} / \mathrm{min} / \mathrm{m}^{3}$. The World Diabetes Atlas showed that the current number of diabetic patients worldwide is about 463 million, and it is estimated to increase to nearly 700 million in 2045 . In 2013, the prevalence rate of diabetes in adults in China was $10.9 \%$, and the prevalence rate of pre-diabetes was $35.7 \%$ and the number of pre-diabetes patients was 388 million. With such a large diabetic population and such a high incidence of kidney involvement, it is foreseeable that diabetic nephropathy will inevitably metamorphose into a major challenge facing the medical community that battles CKD and in the formulation of strategies that are aimed at its prevention and control. It is therefore of imminent necessity to strengthen the prevention of, and develop and institute control measures that are aimed at tackling, diabetic nephropathy.

With an emphasis on the prevention and control of diabetic nephropathy, more and more researches focus on this field, which includes not only the epidemiology and clinical multi-center research of diabetic nephropathy but also the in-depth basic research that aims to explore the mechanism of diabetic nephropathy, screen new biomarkers, and establish novel therapeutic targets. So far, the researchers in the field of diabetic nephropathy have made major progress and continuous breakthroughs and thus provided a series of results. Moreover, the quantity and quality of published articles continue to rise. However, there is currently no professional journal in this field worldwide. Health promotion is the common responsibility of the international community, and diabetic nephropathy has inevitably been the main challenge that requires addressal in the study of diabetes' management and the main direction required of CKD prevention and treatment. Therefore, it is of immediate importance to formulate and launch a specialized periodical to lead the international research front in the field of diabetic nephropathy and show the latest academic progress in its basic science, clinical medicine, big data, and artificial intelligence. The first professional journal of Diabetic Nephropathy in this field was born under this background.

This special issue was co-edited by Professor Danesh Farhad R, Professor Mark E. Cooper, Professor Chen Jianghua, Chairman of the Chinese Society of Nephrology, and Professor Liu Zhangsuo, Secretary-General of the Chinese Society of Nephrology. In the meantime, the editorial board members of this journal hail from famous universities and institutes all over the world. Their profound attainments and international academic influence in the field of diabetic nephropathy research will certainly ensure the scientific nature and sustainable development of this journal.

This journal mainly publishes reviews of domestic and foreign experts in the field of diabetic nephropathy, special 
review, basic research, translational medicine, precision medicine papers, clinical guidelines, clinical consensus, case reports, short stories, letters from readers, technical progress, and other academic articles. In addition to strictly following the international editorial and publishing norms and founding strategies, the journal will also be established and run according to the international high-level journal standards, aiming to publish the latest progress on the subject and lead the academic research directions of diabetic nephropathy. After intensive preparation and coordination, the first issue of the journal will be published shortly. This issue publishes the latest research from nephrologists and their teams all over the world, including basic research, clinical research, research progress review, and case reports. I hope you will find it interesting to through the first issue and find something useful in it. We would love to receive your feedback. We also invite the contributors to actively communicate with our readers, and likewise welcome the readers to contribute actively!

The coronavirus disease 2019 (COVID-19) epidemic is still spreading around the world, posing a huge threat not only to the lives and health of people at large but also to global public health security. No country is immune to disease. More than ever, in the present-day scenario the international community needs solidarity and cooperation to jointly build a safe community for human health. Therefore, it is hoped that we can form a global research upsurge on diabetic nephropathy to raise awareness of this disease among people. It is also expected that the latest research results can be translated into clinical application to benefit more diabetic nephropathy patients.

Over time, we hope that Diabetic Nephropathy will publish more and more influential articles in the field of diabetic nephropathy and provide an indispensable resource for basic researchers and clinicians. Here, on behalf of the editorial and expert team of this journal, I would like to express my heartfelt thanks to my colleagues and friends for their support for our journal. It takes a spirit of adventure to contribute to a new magazine, and we are eternally grateful to you for giving us the opportunity! Best wishes to Diabetic Nephropathy!

\section{REFERENCES}

[1] Zhang L, Wang F, Wang L, Wang W, Liu B, Liu J, cross-sectional survey. Lancet (London, England) 2012; 379: et al. Prevalence of chronic kidney disease in China: a 815-22. 